University of Nebraska - Lincoln

DigitalCommons@University of Nebraska - Lincoln

1966

\title{
Analog Plotting System for Recording Energy Spectra of Low Energy Charged Particles
}

M. Eugene Rudd

University of Nebraska - Lincoln, erudd@unl.edu

Follow this and additional works at: https://digitalcommons.unl.edu/physicsrudd

Part of the Physics Commons

Rudd, M. Eugene, "Analog Plotting System for Recording Energy Spectra of Low Energy Charged Particles" (1966). M. Eugene Rudd Publications. 42.

https://digitalcommons.unl.edu/physicsrudd/42

This Article is brought to you for free and open access by the Research Papers in Physics and Astronomy at DigitalCommons@University of Nebraska - Lincoln. It has been accepted for inclusion in M. Eugene Rudd Publications by an authorized administrator of DigitalCommons@University of Nebraska - Lincoln. 


\title{
Analog Plotting System for Recording Energy Spectra of Low Energy Charged Particles* +
}

\author{
M. E. RUDD
}

Department of Physics, University of Nebraska, Lincoln, Nebraska 68508

(Received 7 March 1966; and in final form, 25 March 1966)

$\mathrm{D}$

URING recent measurements ${ }^{1}$ of energy spectra of electrons emitted from gases under positive ion bombardment an analyzing and plotting system was developed which has some unique features. Utilizing an electrostatic analyzer, it sweeps over the desired energy range and plots a graph directly in electron volts. With suitable modifications it could also be used for positive ions. Energies from near 0 to about $1000 \mathrm{eV}$ have been plotted but this range could be extended. Usable graphs have been obtained at counting rates as low as 3 counts/sec.

Compensation is provided for fluctuations in the signal due to slow or rapid variations in the beam current. The detection system counts individual particles for greatest sensitivity although the recording apparatus could also be adapted to current measurements.

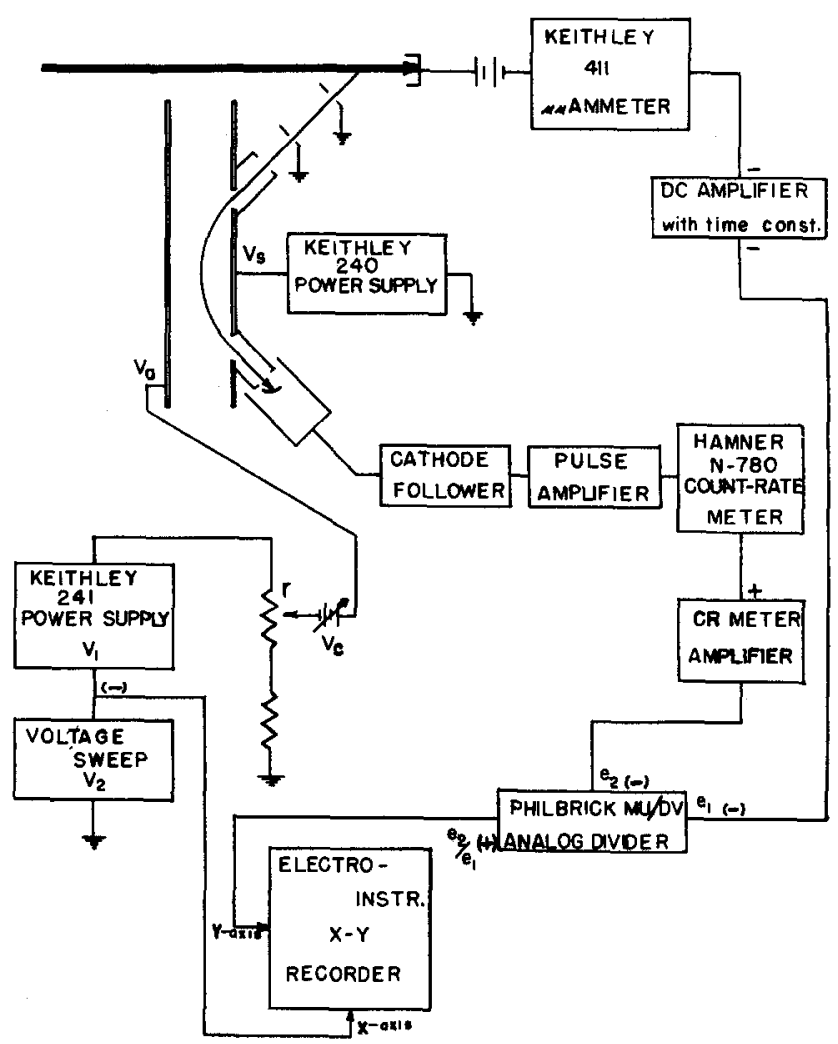

FIG. 1. Block diagram of apparatus. The ion beam (heavy line) passing through a gas ejects electrons which pass through a parallel plate analyzer and are detected by an electron multiplier. The voltage $V_{a}$ on the back plate is varied, thus passing electrons of varying energies. The counting rate meter signal is divided by the beam current signal and the ratio is plotted on the recorder against electron energy. 
Variations in counting rate due to beam current fluctuations are taken care of by dividing the counting rate by the beam current. In a plotter described by Briglia and Rapp ${ }^{2}$ this division is performed by the use of an external recorder reference voltage proportional to the beam current. In the present system the division is performed by a commercial analog divider. While this is a slightly more complicated system it has the advantage that it does not require the use of a floating electrometer and a floating input dc amplifier nor is it necessary to make any internal connections to the $\mathrm{X}-\mathrm{Y}$ recorder. The cost of the components is comparable. Since we are looking for fine structure in the energy spectra a continuous plot was desired rather than the digital type plot obtained by Briglia and Rapp.

A block diagram of the apparatus is given in Fig. 1. A parallel plate electrostatic analyzer of the type described by Harrower ${ }^{3}$ is used. The front and back plates are supplied with voltages from separate supplies. The front plate is kept at a constant potential for any one run while the back plate is supplied by a varying sweep voltage which covers the energy range of interest. The variable part of this back plate voltage is also fed to the $\mathrm{X}$ axis of the $\mathrm{X}-\mathrm{Y}$ recorder.

If the constant of the analyzer is defined to be the ratio of the potential difference between the plates to the energy of electrons as they enter the analyzer, then Harrower showed ${ }^{3}$ that this constant is given by $c=2 d / x$, where $d$ is the plate spacing and $x$ is the separation of the centers of the slits. Then if $V_{a}$ and $V_{s}$ are the potentials of the back and front plates, respectively, the energy of the charged particles passed by the analyzer is $E=q\left[V_{a} / c+(c-1)\right.$ $\left.\times V_{a} / c\right]$, where $q$ is the charge of the particles. This equation takes account of the pre-acceleration of the particles by the potential $V_{s}$ on the front plate. This pre-acceleration voltage is used to vary the analyzer resolution since the width of the resolution curve is proportional to the energy of the particles as they enter the analyzer. This energy can be increased or decreased by choosing $V_{\mathrm{s}}$ properly.

Since it was desired to plot electron energies directly on the $\mathrm{X}$ axis one requires $E=q\left(V_{1}+V_{2}\right)$, where $V_{1}$ is the baseline voltage and $V_{2}$ is the sweep voltage. The voltage applied to the back plate is given by $V_{a}=r\left(V_{1}+V_{2}\right)+V_{c}$, where $r$ is the voltage divider ratio (see Fig. 1) and $V_{c}$ is a small compensating voltage. Combining the last three equations it is seen that in order to plot electron energies directly on the plotter the following conditions must be met:

$$
\begin{aligned}
r & =c, \\
V_{c} & =(1-c) V_{s .} .
\end{aligned}
$$

The plate spacing of the analyzer was adjusted to make $c$ about $\frac{1}{2} \%$ less than unity. Then the final values of $r$ and
$V_{c}$ were set using an electron gun with an accurately known beam energy. The value of $r$ was set using a fairly high electron energy and the value of $V_{c}$ was set at a low electron energy.

The voltage sweep circuit consists of a voltage source and a resistor network which supplies a constant current to a polystyrene capacitor connected across an operational amplifier to form a current integrator. The output of the amplifier provides the desired sweep voltage. The sweep rate can be set by adjusting the current to the integrator.

Since the counting rate meter has only a $0.1 \mathrm{~V}$ output this is amplified by a gain of $100 \mathrm{dc}$ amplifier. The output of the electrometer reading the beam current is also amplified. A time constant is incorporated into this amplifier, the value of which has to be matched to that of the counting rate meter in order that transient changes of the beam current would not cause transients in the output of the divider.

The analog divider is a Philbrick model HM operational manifold. This consists of one of the dual units from the MU/DV multiplier-divider plus five sockets for plug-in operational amplifiers. The voltage $e_{1}$ to the divider must be negative and for accuracy should be as large as possible

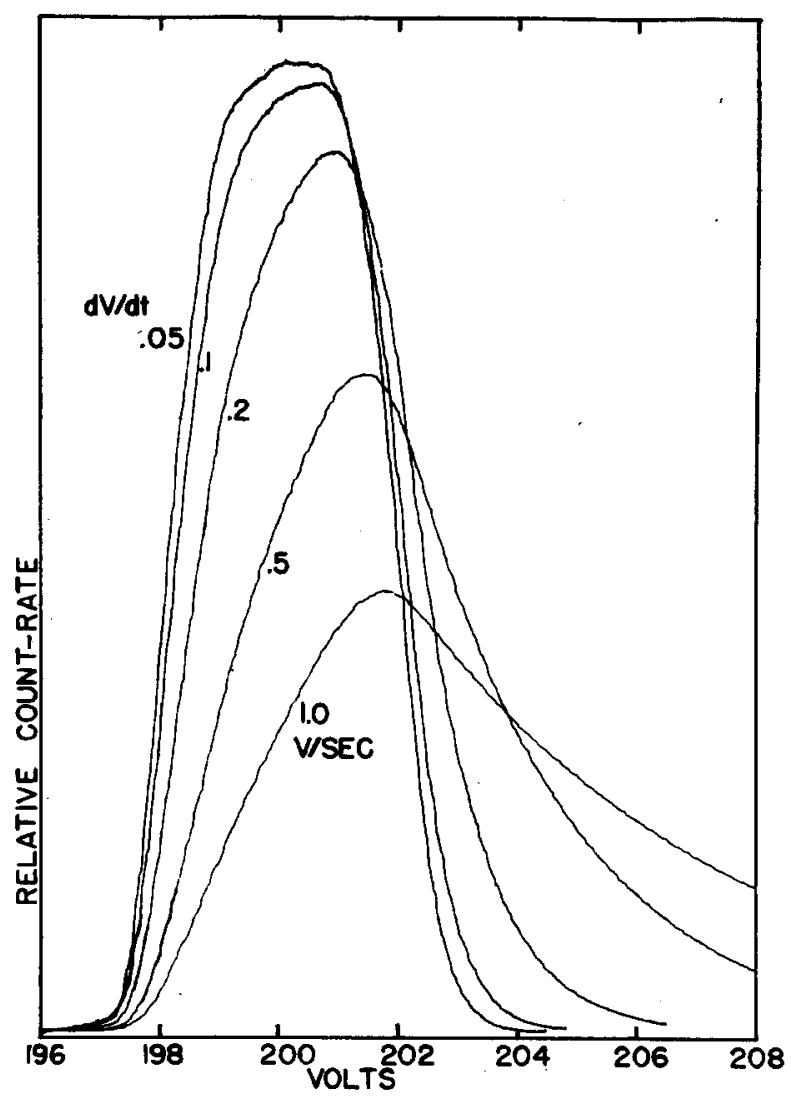

FIG. 2. Effect of too rapid voltage sweep. Maximum counting rate was about 9000 counts/sec, time constant $4 \mathrm{sec}$, and sweep rates as marked on curves. 
up to $50 \mathrm{~V}$. The components in the amplifiers were chosen to satisfy this condition.

The time constants in both channels are set according to the average counting rate $n$ and the desired fractional probable error $e$. The relation between these quantities is given by Elmore and Sands ${ }^{4}$ and can be written approximately $T_{c}=2 /\left(9 n e^{2}\right)$. The sweep rate is set after the time constant is selected. If the sweep rate is too great there is an undesirable change in the shape of the resolution peak characterized by a decrease in peak height, a shift of the center of the peak, and a decrease in the resolution. This effect is illustrated in Fig. 2. To minimize this effect the sweep rate is chosen by the relation $d V / d k \ll$ FWHM $/ T_{c}$ where FWHM is the full width at half maximum of the analyzer resolution curve. In practice, the inequality is well enough satisfied by a factor of five for most purposes. This corresponds approximately to the curve of $d V / d t=0.2$ $\mathrm{V} / \mathrm{sec}$ in Fig. 2. The shift of the center of the peak is given approximately by $\Delta V=T_{c}(d V / d t)$ and compensation can thus be made for this shift for accurate work.

When the divider is adjusted in accordance with the manufacturer's instructions it is possible to vary the input beam current by a factor of 10 without varying the divider output more than about $2 \%$.

As pointed out by Harrower ${ }^{3}$ the resolution FWHM of the analyzer is proportional to the energy of the electrons as they enter the analyzer. In order not to distort the energy distributions he suggests a correcting circuit which changes the gain of the output signal in inverse proportion to the energy of particles passing through the analyzer. We have occasionally used a different method of compensating for this effect. Instead of holding the front plate of the analyzer at a constant potential, the potential difference between the plates is kept constant while the back plate potential is varied. The effect of this is to accelerate (or decelerate) all the charged particles of the energies to be detected to the same energy before they enter the analyzer. This keeps the resolution (FWHM) constant as the energy range is swept. If the analyzer constant $c$ is made very nearly unity, then Eq. (2) can be satisfied (by setting $V_{c}=0$ ) even though $V_{\varepsilon}$ now varies.

It is a pleasure to acknowledge the excellent suggestions made by C. E. Kuyatt, and the help of C. A. Sautter, D. Lang, D. Gregoire, and G. Sandness in the construction and testing.

* Work supported by the National Science Foundation.

† The initial work on this project was done at Concordia College, Moorhead, Minnesota $\mathbf{5 6 5 6 0 .}$

${ }^{1}$ M. E. Rudd, Phys. Rev. Letters 13, 503 (1964); and 15, 580 (1965).

2 D. D. Briglia and D. Rapp, Rev. Sci. Instr. 36, 1259 (1965).

${ }^{3}$ G. A. Harrower, Rev. Sci. Instr. 26, 850 (1955).

${ }^{4}$ W. C. Elmore and M. Sands, Electronics (McGraw-Hill Book Company, Inc., New York, 1949), p. 252. 